\title{
Acoustic emission and fluctuations of electroluminescence intensity in light-emitting heterostructures
}

\author{
V.P. Veleschuk ${ }^{1}$, O.V. Lyashenko ${ }^{2}$, Z.K. Vlasenko ${ }^{1}$, M.P. Kysselyuk ${ }^{1}$ \\ ${ }^{1} V$. Lashkaryov Institute of Semiconductor Physics, NAS of Ukraine, \\ 41, prospect Nauky, 03028 Kyiv, Ukraine \\ ${ }^{2}$ Taras Shevchenko Kyiv National University, \\ 2, Academician Glushkov Ave., 03022 Kyiv, Ukraine \\ E-mail:lyashenk@univ.kiev.ua
}

\begin{abstract}
It was shown that in the $\mathrm{GaAsP} / \mathrm{GaP}$ and $\mathrm{InGaN} / \mathrm{GaN}$ heterostructures during current passage redistribution of electroluminescence intensity on the structure surface takes place simultaneously with radiation of acoustic emission. Local (on surface area) fluctuations of electroluminescence intensity are observed together with general degradation of electro-physical parameters.
\end{abstract}

Keywords: acoustic emission, light-emitting diodes, electroluminescence.

Manuscript received 05.10.09; accepted for publication 22.10.09; published online 30.12.09.

\section{Introduction}

Evolution with time and change of current of nonuniform distribution of electroluminescence (EL) intensity on radiating surface of light-emitting diodes (LEDs) based on the InGaN/GaN and $\mathrm{GaAsP} / \mathrm{GaP}$ heterostructures was already described by us $[1,2]$ and many other researchers, for example in [3-6]. Active processes of defects formation and fluctuation in these heterostructures in excess of the density of direct current $(J)$, which is specified by the manufacturer as maximal density of the direct current $J_{\max }$, impose the certain restrictions on their field of application, because EL intensity could be several times higher with $J$ increase $[1,2,7-10]$. A similar possibility for the pulse current (pulse duration 3 and $0.3 \mu$ s, off-duty factor $\sim 3 \cdot 10^{3}$ ) was shown in [11].

To solve these problems, many physical mechanisms of the active processes, in particular, dynamics of defects development, transformation of functional parameters of LEDs and also their interrelation should be investigated [1-12].

Increase of the integral EL intensity $I$ in LEDs, caused by injection current density $J$ increase, leads to non-uniform distribution of $I$ [3-10] on the active area of the structure and on any cross-section of the current tube [8-10], in particular, due to effect of current crowding [3, 5], and also to significant additional local overheating of the active area $[1,2,5-10]$. These and other factors considerably decrease LEDs efficiency.
In the InGaN/GaN LED active area, the gradient $\Delta T(r) / \Delta r$ reaches a considerable value (by our estimations not less than $10^{3}-7 \cdot 10^{3} \mathrm{C} / \mathrm{cm}[1,10]$, according to $\left.[5,12]-\sim 10^{4}-2 \cdot 10^{5} \mathrm{C} / \mathrm{cm}\right)$. This fact, taking into account distinction of the thermal expansion coefficients $\alpha_{i}$ and lattice constants $a_{i}$ of individual layers, leads to formation of local inelastic thermomechanical stresses in $p$ - $n$ junction $[1,2,5,7-10,12]$. At the same time, it is known that ultra-fast relaxation of these induced mechanical stresses in LEDs results in appearance of spontaneous chaotic acoustic radiation acoustic emission (AE) of materials [1, 2, 7-10, 13].

The purpose of this work was to analyze dynamics of local processes in relaxation and defects formation in the light-emitting InGaN/GaN and $\mathrm{GaAsP} / \mathrm{GaP}$ heterostructures, induced by the direct current, which leads to simultaneous changes in the EL intensity distributions on a surface of the structure $I(S)$, AE occurrence, oscillation of $I$, and also fluctuations of current and general degradation of electro-physical parameters of heterostructures.

\section{Experimental}

Research of correlation of AE, fluctuations of EL intensity $\Delta I$ and current was carried out using technique described in $[1,2]$. AE signals were registered by the piezoelectric transducer of the specialized acousticemission device AF-15, integrated $I$ was registered by photodiodes. These signals and current noises were 
processed by ADC. Objects of researches were lowsized $(\mathrm{GaAsP} / \mathrm{GaP}) \quad$ (Fig. $1 \mathrm{~b}, \mathrm{~d})$ and nanosized (InGaN/GaN) (Fig. 1b, d, f) structures.

The EL intensity distribution at surface $I(S)$ (Fig. 1a, c and Fig. 2a, c, e), and along the selected section (Figs 3 and 4) were obtained by frame-by-frame computer analysis of digital video (or directly from original digital photos) of the structure surface taken using microscope with zoom $\times 98$.

\section{Results}

In Fig. 1a, c distributions $I(S)$ for the GaAsP/GaP heterostructure are presented in the spectral range 410$700 \mathrm{~nm}$ at different injected currents. It is seen that an increase of intensity distribution $I(S)$ with current becomes more heterogeneous on the surface - intensity is concentrated near electrode, and on edges of the structure intensity decreases that can be connected with decrease of the $p$ - $n$ junction resistance, relative decrease of the lateral component of the current and, probably, with crowding of the current between electrodes [3-5]. Similar results were obtained for $\mathrm{InGaN} / \mathrm{GaN}$ heterostructures.

In Fig. 2a, c, e $I(S)$ of the $\mathrm{InGaN} / \mathrm{GaN}$ heterostructure in the same spectral range measured in an orderly sequence at $80 \mathrm{~ms}$ interval are presented. In the process changes of the intensity in separate local areas of the structure surface $\Delta I\left(S_{i}\right)$ were observed during AE. Similar results were obtained for $\mathrm{GaAsP} / \mathrm{GaP}$ heterostructures.
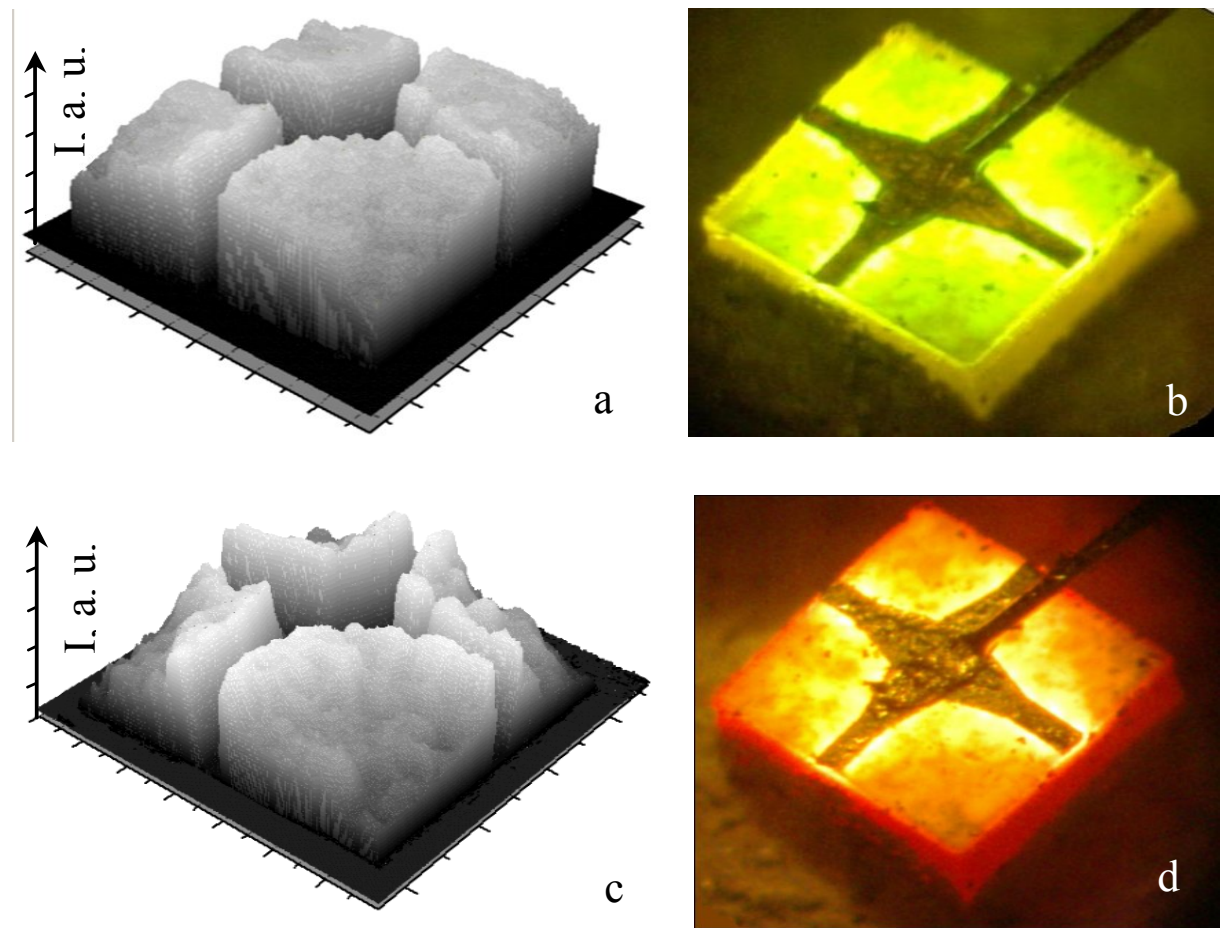

Fig. 1. EL intensity surface distribution $I(S)$ for the $\mathrm{GaAsP} / \mathrm{GaP}$ heterostructure at $68 \mathrm{~A} / \mathrm{cm}^{2}$ (a) and $172 \mathrm{~A} / \mathrm{cm}^{2}$ (c) and their micrographs $(b, d)$. 

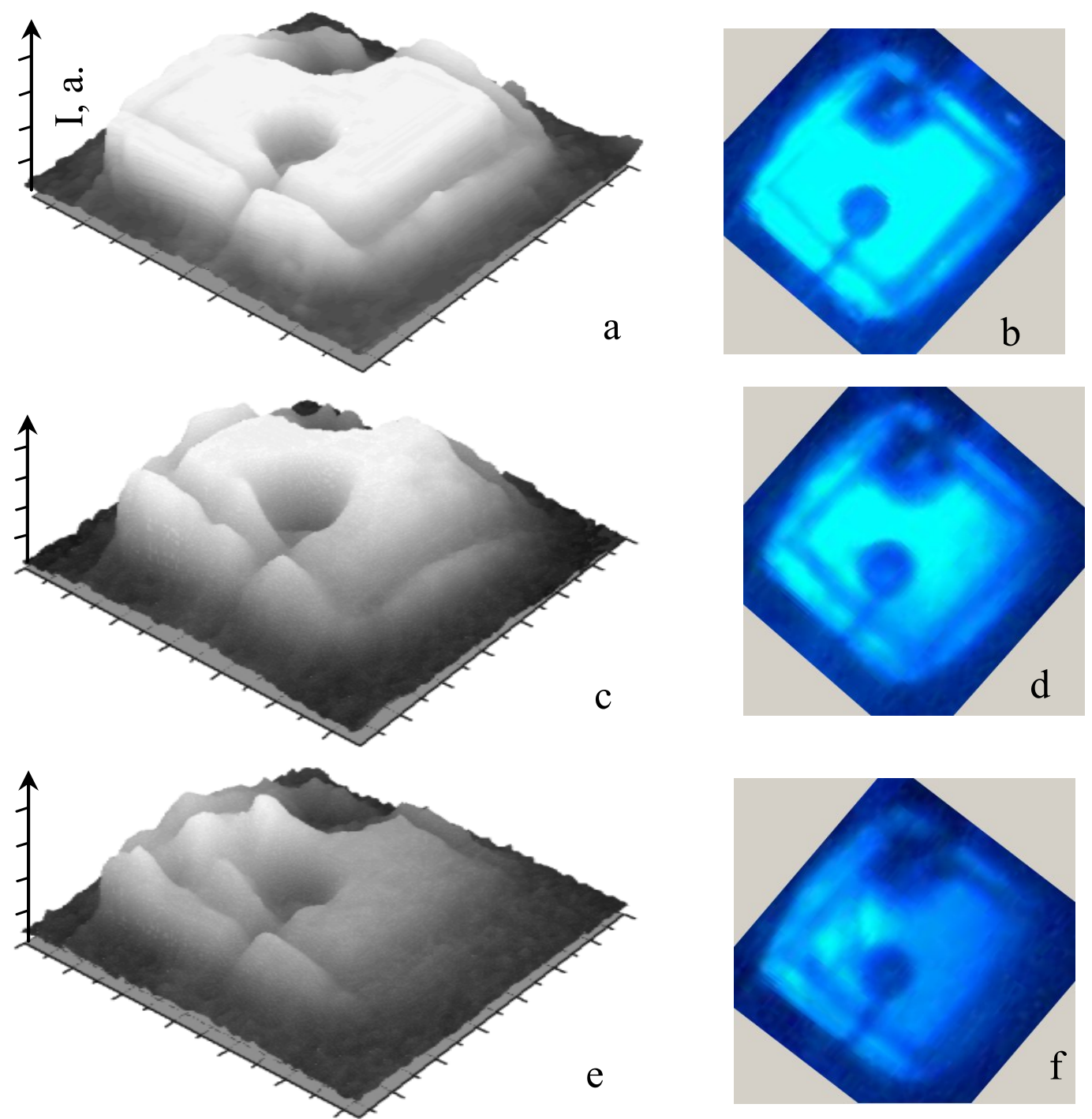

Fig. 2. EL intensity surface distribution $I(S)$ for the $I n G a N / G a N$ heterostructure at $110 \mathrm{~A} / \mathrm{cm}^{2}$ at sequential moments (a, c, e) and their micrographs $(b, d, f)$.

characteristics (CurVC) and capacitance-voltage characteristics (CVC) took place, which were similar to those described by us earlier in [10].

\section{Discussion}

One of the problem of fabrication and operation of these structures, in which the average value of internal mechanical stresses reaches $\sim 10^{9} \mathrm{~Pa}$ [2], is local heterogeneities in conductivity, thermal resistance, difference of elastic modules [1, 2, 7-10], and also $\alpha_{i}$ and $a_{i}$, mentioned above. At current passage, it leads to formation of the temporary additional local thermomechanical stresses $\left(>10^{7}-10^{8} \mathrm{~Pa}\right)$, which are distributed in unpredictable manner (chaotically) in the structure bulk and on its interfaces, areas and lateral surfaces adjoining them due to a complex spatial distribution of the temperature gradients.
The complex of these reasons leads to individual dynamics of degradation in local areas of the heterostructures. Degradation and fluctuations of electrophysical properties and integrated EL intensity [1, 2, 710] as well as the current noises of the locally nonuniform thermo-stressed heterostructures, simultaneous with $\mathrm{AE}$, are integrated manifestations of this fact.

Defects formed or changed their state in the active area due to formation of additional system of the energy levels in the area of hetero-junction act as both the additional scattering centers of the charge carriers and their tunneling centers. At $J$, exceeding AE appearance threshold, $I$ decreases and changes in separate local areas because of activation of defects-formation processes, thus a redistribution of relative $I(S)$ on the structure surface takes place.

Intensive appearance of the structural defects as well as changes in their energy state, accompanied by

\section{(C) 2010, V. Lashkaryov Institute of Semiconductor Physics, National Academy of Sciences of Ukraine}


AE, lead to local $\Delta J_{i}$ fluctuations due to fast local changes of heterostructure resistance. In turn, $\Delta J_{i}$ leads to fluctuations of carriers injection into the quantum well, which recombine there with radiation. It causes fluctuations $\Delta I\left(S_{i}\right)$ in local areas of radiating surface $S$, and appearance of the dependences $I$ and $\Delta I$ on $S$ and $t$ $I(S, t)$ and $\Delta I(S, t)$.

Let us note that the factors influencing the dependences $I(S, t)$ and $\Delta I(S, t)$ are also temporary local appearance of conductive channels (shunting $p-n$ junction) and their subsequent breakings $[4,6]$. Fluctuations of recombination current due to redistribution of recombination and tunnel components of current, caused by growth of leakage current at crossing of the $p-n$ junction area by defects (dislocations) and decrease of injection factor due to capture of carriers on the traps at defects formation in the field of contacts are also possible. In paper [6], fluctuations were observed in $\mathrm{GaN}$ resonant-cavity LEDs, which correlated with CurVC changes that also corresponds to our researches $[2,8,10]$. Authors of [6] explain this effect by metal atoms migration along threading dislocations and nanopores.
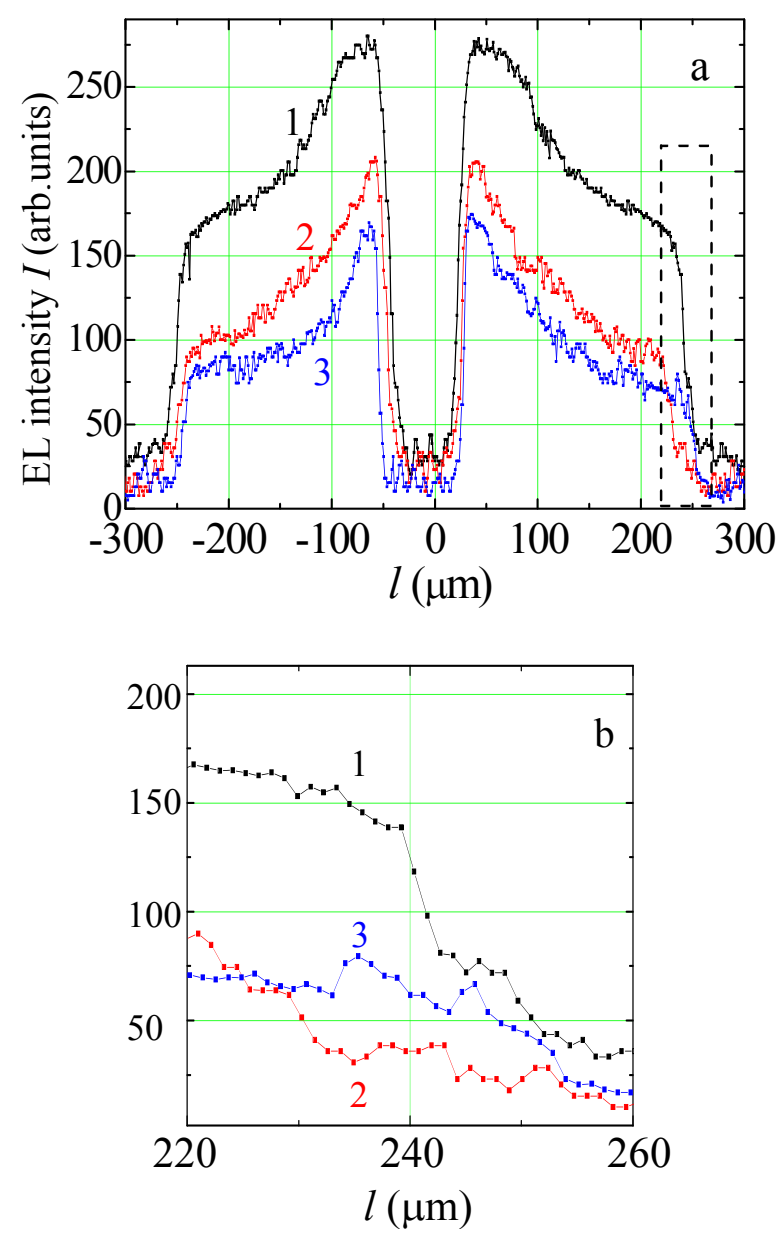

Fig. 3. Distribution $I(l): 1-J=80 \mathrm{~A} / \mathrm{cm}^{2} ; 2-140 \mathrm{~A} / \mathrm{cm}^{2} ; 3-$ $80 \mathrm{~A} / \mathrm{cm}^{2}$ after $\mathrm{AE}$ (a) and selected area (b) from (a).

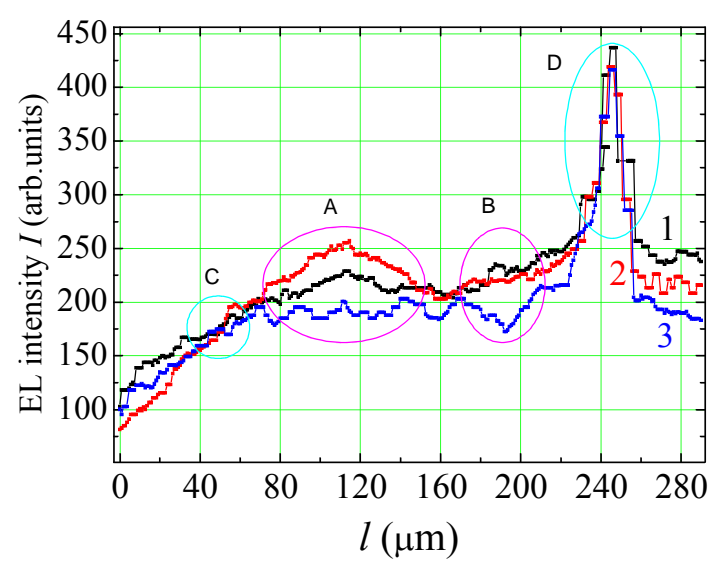

Fig. 4. Distribution $I(l): 1-\mathrm{t}=0,2-\mathrm{t}=40 \mathrm{~ms}, 3-\mathrm{t}=80 \mathrm{~ms}$; $J=110 \mathrm{~A} / \mathrm{cm}^{2}$.

Shown by us (see Figs 1-4) non-uniform distribution of EL intensity and formation of local areas with unique dynamics of degradation correlates well with definition of AE source as area of object under investigation in which transformation of any form of energy into mechanical energy of AE [13] takes place. Thus, defects formation processes accompanying with $\mathrm{AE}$ radiation can result in fluctuations of EL intensity on LED radiating surface.

There are at least two ways of degradation (change of the efficiency of the "quantum yield") depending on changes in the defect structure of light-emitting heterostructure. In one of them, chaotic vibrationaltranslatory motions of the single dislocations, which cause appearance of continuous low-energy AE, do not change essentially a "degree of deficiency" of the structure, i.g., the density of dislocations remains approximately at the initial level. Vibrational-translatory motion of dislocations in this case is only a part of the mechanism of super-fast relaxation of excessive local mechanical stresses accompanying by $\mathrm{AE}$ occurrence and, consequently, determines only redistribution of $I(S)$ and appearance of $\Delta I(S)$.

Other way supposes substantial increase of both dislocations density and three-dimensional defects, and, consequently, irreversible individual reduction for each $I\left(S_{i}\right)$ area and also $\Delta I\left(S_{i}\right)$ appearance.

Distribution $I(S)$ in Fig. 1 corresponds to the first of described ways, and distribution in Fig. 2 corresponds to the second one.

\section{Conclusion}

It was shown that at current increase in the $\mathrm{GaAsP} / \mathrm{GaP}$ and $\mathrm{InGaN} / \mathrm{GaN}$ heterostructures not only integrated but also local degradation of the electroluminescence intensity takes place simultaneously with acoustic emission appearance. Thus, evolution of electroluminescence intensity surface distribution, which is related with integrated degradation of other electro-physical characteristics, including CurVC and $\mathrm{CVC}$, can bear 
witness to local changes of electro-physical characteristics in surface and subsurface layers of the heterostructures.

\section{References}

1. O.V. Lyashenko, V.P. Veleshchuk, O.I. Vlasenko, R.G. Chuprina, Dynamics and Time Correlation of Acoustic Emission, Electrical Noises and Quantum Yield Fluctuations in Optoelectronic Devices // AIP Conference Proc. 922, p. 216-222 (2007).

2. A.I. Vlasenko, O.V. Lyashenko, P.F. Oleksenko, and V.P. Veleschuk, Fluctuations of current, electroluminescence and acoustic emission in lightemitting $\mathrm{A}^{3} \mathrm{~B}^{5}$ heterostructures // Semiconductor Physics, Quantum Electronics \& Optoelectronics 11(3), p. 230-235 (2008).

3. X. Guo and E.F. Schubert, Current crowding in $\mathrm{GaN} / \mathrm{InGaN}$ light emitting diodes on insulating substrates // J. Appl. Phys. 90(8), p. 4191-4195 (2001).

4. N.I. Bochkareva, A.A. Efremov, Yu.T. Rebane, R.I. Gorbunov, A.V. Klochkov, and Yu.G. Shreter, Nonuniformity of carrier injection and degradation of blue LEDs // Fizika i tekhnika poluprovodnikov 40(1), p. 118-123 (2006), in Russian.

5. A.V. Zinovchuk, Effect of current crowding on the behavior of electron-hole plasma in multilayer light emitting structures // Thesis for the Degree of Candidate of Phys.-Math. Sci., spec. 01.04.07, Kyiv, p. 20 (2008).

6. B. Roycroft, M. Akhter, P. Maaskant, B. Corbett, A. Shaw, L. Bradley, P. de Mierry, and M.-A. Poisson, Origin of power fluctuations in GaN resonant-cavity light-emitting diodes // The international electronic journal of optics "Optics Express” 12(5), p. 736-741 (2004).
7. V.P. Veleshchuk and O.V. Lyashenko, Acoustic emission of light-emitting structures on the $\mathrm{A}^{3} \mathrm{~B}^{5}$ base determined by direct current // Ukrainsky Fizychny Zhurnal 48(9), p. 981-985 (2003), in Ukrainian.

8. V.P. Veleschuk, O.I. Vlasenko, O.V. Lyashenko, Yu.O. Myagchenko et al., Acoustic emission on the relaxation of local thermomechanical stresses in the process of degradation of light-emitting heterostructures on the basis of InGaN and GaAsP // Ukrainian Journal of Physics 53(3), p. 240-246 (2008).

9. V.P. Veleshchuk, O.V. Lyashenko, Yu.A. Myagchenko, and R.G. Chuprina, Evolution of electroluminescence spectra and the acoustic emission of epitaxial structures GaAsP // J. Appl. Spectroscopy 71(4), p. 553-557 (2004).

10. V.P. Veleschuk, O.I. Vlasenko, O.V. Lyashenko, A. Baidullaeva, B.K. Dauletmuratov, Acoustic emission and changes of luminescent and electrical characteristics of $\mathrm{InGaN} / \mathrm{GaN}$ heterostructures at the current loading // Physics and Chemistry of Solid State 9(1), p. 169-174 (2008).

11. A.A. Efremov, N.I. Bochkareva, R.I. Gorbunov, D.A. Lavrinovich, Y.T. Rebane, D.V. Tarkhin, and Y.G. Shreter, Influence of Joule heating on quantum efficiency and heat regime options of high power blue InGaN/GaN light-emitting diodes // Fizika i tekhnika poluprovodnikov 40(5), p. 621627 (2006).

12. A.A. Bergh and P.J. Dean, Light Emitting Diodes. Mir, Moscow, 1979 (in Russian).

13. V.A. Greshnikov and Yu.B. Drobot, Acoustic Emission. Izd-vo Standartov, Moscow, 1976 (in Russian). 